\title{
The impact of infection on host competition and its relationship to parasite persistence in a Daphnia microparasite system
}

\author{
Dominik Refardt • Dieter Ebert
}

Received: 20 December 2010/Accepted: 6 May 2011/Published online: 20 May 2011

(C) Springer Science+Business Media B.V. 2011

\begin{abstract}
Evolutionary studies often estimate fitness components with the aim to make predictions about the outcome of selection. Depending on the system and the question, different fitness components are used, but their usefulness for predicting the outcome of selection is rarely tested. Here we estimate host fitness components in different ways with the aim to test how well they agree with each other and how well they predict host fitness at the population level in the presence of the parasite. We use a Daphnia magna-microparasite system to study the competitive ability of host clones in the absence and presence of the parasite, the infection intensity of the parasite in individuals of twelve host clones (an estimate of both host resistance and parasite reproductive success), and parasite persistence in small host populations (an estimate of $R_{0}$ of the parasite). Analysis of host competitive ability and parasite persistence reveals strong host genotype effects, while none are found for infection intensity. Host competitive ability further shows a genotype-specific change upon infection, which is correlated with the relative persistence of the parasite in the competing hosts. Hosts in which the parasite persists better suffer a competitive disadvantage in the parasite's presence. This suggests that in this system, parasite-mediated selection can be predicted by parasite persistence, but not by parasite infection intensity.
\end{abstract}

Keywords Competition · Fitness components · Red Queen · Resistance · Virulence

D. Refardt $\cdot$ D. Ebert

Département de Biologie, Unitè Ecologie and Evolution, Université de Fribourg, chemin du Musée 10, 1700 Fribourg, Switzerland

D. Refardt (西)

Institut für Integrative Biologie, ETH Zürich, Universitätstrasse 16, CHN J 11, 8092 Zürich, Switzerland

e-mail: dominik.refardt@gmail.com

Present Address:

D. Ebert

Zoologisches Institut, Universität Basel, Vesalgasse 1, 4051 Basel, Switzerland 


\section{Introduction}

The fitness of genotypes, i.e. their contribution to future generations, although a key variable in evolutionary biology, can be difficult if not impossible to measure in empirical studies. Fitness components, traits that contribute to fitness, are therefore often used to make inferences about total fitness. Because this shortcut bears the danger of introducing a bias, any inferences must be made with appropriate caution (Rolff and Joop 2002). Different fitness components can combine multiplicatively to total fitness and the detection of among-genotype variation can fail with the ignorance of a key component (SchmidHempel and Ebert 2003). Likewise, trade-offs among components, e.g. between fecundity and longevity (Stearns 1992), can render the explanatory power of an isolated component useless. That fitness components provide an appropriate estimation of total fitness is often assumed yet rarely tested.

This issue is further complicated in host-parasite systems, where it is important not only to accurately measure host and parasite fitness, but also to correctly establish the relationship between them. Assumptions about this relationship form the basis of important hypotheses on the maintenance of genetic diversity, local adaptation, evolution of sex and recombination, and evolution of parasite virulence (Clarke 1979; Hamilton 1980; Hamilton and Zuk 1982; Bull 1994; Thompson 1994; Dieckmann 2002). Due to their intimate relationship, it is often difficult to assign a trait unambiguously to either the host or the parasite. For example infection intensity, the number of parasites that builds up in an infected host, is thought to correlate positively with the parasite's transmission success. Thus, it may serve as an estimator of parasite fitness. At the same time, infection intensity is related to the host's ability to restrict parasite growth and may therefore be negatively correlated with host fitness.

The relationship between host and parasite fitness is condensed in notions such as virulence, the increased host mortality resulting from parasite infection (May and Anderson 1983; Bull 1994), or resistance, the ability to prevent infection or limit its extent (Roy and Kirchner 2000; Rigby et al. 2002). The 'trade-off' hypothesis states that parasite virulence is the necessary consequence of parasite replication within a host (Frank 1996). Parasites must harm their host in order to ensure their own transmission, and virulence is therefore positively correlated with parasite fitness up to an optimal level of virulence (Anderson and May 1982). This view of virulence appears to be bolstered by numerous observations where parasites evidently harm their hosts to advance in their life cycle. Yet despite the intuitive appeal of this view, there are only a few cases where the appropriate empirical data are available that allow evaluation of the hypothesis (Mackinnon and Read 1999; Jensen et al. 2006; de Roode et al. 2008).

An analogous situation exists for resistance, which also links host and parasite fitness (Grech et al. 2006). Increasing levels of resistance will decrease a parasite's transmission success and should cause a negative relationship between host and parasite fitness. However, the unequivocal estimation of this relationship must take into account any costs, which must be subtracted to obtain the net benefit gained by the host (Schwarzenbach and Ward 2006; Vijendravarma et al. 2009). Clearly, an estimation of virulence or resistance that takes its evolutionary consequences into account must be based upon independent fitness measurements of both host and parasite.

The experiments presented here attempt to establish the relationship between the fitness of different genotypes of the freshwater crustacean Daphnia magna and an isolate of a gut microparasite (Ebert 2005). We measured host fitness as intraspecific competitive ability under food limited conditions in pairwise competitions of clones both in the presence and 
absence of the parasite. Competitive ability summarizes several important life-history traits and is a good predictor of fitness in experimental populations (Jungen and Hartl 1979; Little et al. 2002; Capaul and Ebert 2003). Asexual competition is also the predominant mode by which populations evolve during the summer season, which makes competitive ability a realistic estimate for host fitness in this system (Ebert et al. 2002). We estimated parasite fitness individually in all host clones by measuring parasite persistence over several host generations in small experimental populations. This provides a close estimate of the lifetime reproductive success of the parasite, because only parasites with a basic reproductive rate of $R_{0}>1$ can establish persistent infections (Anderson and May 1981; Refardt and Ebert 2007). We also estimated parasite fitness as infection intensity in single hosts, a measure that has been found to correlate with parasite persistence in a previous study with a different microparasite of D. magna, the microsporidian Ordospora colligata (Refardt and Ebert 2007).

\section{Materials and methods}

The host-parasite system

Daphnia magna Straus is a small $(<5 \mathrm{~mm})$ freshwater crustacean of ponds and lakes in Europe. It reproduces by cyclical parthenogenesis, typically with an asexual growth phase during the summer season. Facing unfavourable conditions, Daphnia undergo sexual reproduction, which results in resting eggs. At $20^{\circ} \mathrm{C}$ and good feeding conditions maturity is reached after approximately 10 days and clutches of clonal offspring are released every 3-4 days thereafter. In the laboratory, individual genotypes can be maintained as a population of a single clone. This allows repeated assays on the same genotype as well as competition experiments between genotypes in experimental populations. We kept animals in artificial medium (Ebert et al. 1998), and provided the unicellular green algae Scenedesmus sp. as food supply. With constant food supply, Daphnia populations reach a more or less stable population size and can be kept in this way for many generations.

We used twelve D. magna genotypes, three from each of four origins: Cumnor (near Oxford, UK; genotypes C1-3), Heverlee (near Leuven, Belgium; H1-3), Kniphagen (near Plön, Germany; K1-3), and Ladykirk (80 km southeast of Edinburgh, UK; L1-3).

The parasite used in this study is an isolate of an as yet undescribed unicellular gut parasite from a D. magna population from a carp pond (K35) in Ismaning, north-east of Munich, Germany. The parasite is likely the same species that has been referred to in earlier studies as Microsporidium 1 or Micro1 (Decaestecker et al. 2003, 2005; Fels 2005; Fels et al. 2004) and possibly also Pleistophora intestinalis (Stirnadel and Ebert 1997). The latter name has been used for different, very similar gut parasites of Daphnia in older studies (Larsson et al. 1996). The parasite transmits horizontally via spores and infects epithelial cells of the hindgut of its host. It reproduces intracellularly and forms clusters of spores, which are then released with the faeces. A single infectious cycle lasts only a few days, yet infections are typically chronic because infections quickly disseminate in the gut. The parasite was found at a consistently high $(>50 \%)$ prevalence in a natural population without a discernible effect on host fecundity (Decaestecker et al. 2005). In the laboratory, it reduces lifetime fecundity of its host by $15 \%$, but has no effect on host mortality (Decaestecker et al. 2003).

The parasite isolate was kept in monoclonal populations of its original host genotype. These stocks were used to obtain spores or animals serving as spore donors for infections. 
All experiments were carried out under constant temperature $\left(20 \pm 1^{\circ} \mathrm{C}\right)$ and a $16: 8 \mathrm{~h}$ light:dark cycle. All hosts were cured several months before the experiments by isolation of embryos from the brood pouch of their mother, which prevents infection by horizontally transmitted parasites (Ebert 2005). To verify curing and to check for vertically transmitted parasites, absence of infection was verified by phase contrast microscopy at $400 \times$ magnification and by PCR. The latter used specific primers against parasites known to occur in these clones and general primers against microsporidian parasites, which are a common infection in D. magna (Refardt et al. 2002; Refardt and Ebert 2006, 2007).

Experiment 1: Competition between hosts with and without infection

Host clones were competed pairwise in microcosms for approximately 7 weeks. Host genotypes $\mathrm{C} 1$ and $\mathrm{C} 2$ from Cumnor were competed each against host genotypes H1, H2, $\mathrm{K} 1, \mathrm{~K} 2, \mathrm{~L} 1, \mathrm{~L} 2$ and L3 in the absence and presence of the parasite in four replicates (i.e. 14 pairs $\times 2$ treatments $\times 4$ replicates $=112$ competitions). One replicate competition of $\mathrm{C} 2$ against L1 without parasite was lost.

Prior to competition, animals of each clone were raised in duplicates and one population was exposed to the parasite by adding infected animals within a net enclosure. The net (mesh size $250 \mu \mathrm{m}$ ) kept infected and uninfected animals from mixing, but allowed algae and parasite spores to pass through. Depending on the number of animals required for the competition experiment, containers of different size (4-12 L) were used and feeding and exposure regimes were adjusted accordingly. All clones were kept at a density of 150 animals/L, fed $10^{6}$ cells of algae per animal per day and were exposed to 0.05 infected animals per uninfected animal. Microscopical examination revealed that all exposed populations were infected 7 days post-exposure. Twenty days post-exposure, populations were split into groups of 50 animals with equal size distributions and competitions were started by pooling groups of two clones in a larger jar $(1.3 \mathrm{~L})$. Populations were fed $2 \times 10^{8}$ algal cells every other day, which ensured that they were close to their carrying capacity from the start of the experiment and selection was only for competitive ability under food limited conditions (preliminary experiments showed that a single adult ceases to reproduce when fed less than approximately $10^{6}$ algal cells per day). Infected control populations consisting of single clones were maintained throughout the duration of the experiment.

Competitions lasted between 46 and 52 days, after which changes in clonal frequencies were determined. 72 individuals of every population were sampled randomly and typed by allozyme electrophoresis at the marker locus that was diagnostic for the respective pair (either fumarase, enzyme commission number EC 4.2.1.2, or mannose-6-phosphate isomerase, EC 5.3.1.9). Competitive ability of one competitor relative to the other was estimated for each replicate by a formula linking relative competitive ability to the change in frequency during clonal competition (Hartl 1987): $t \ln (w)=\ln \left(h 1_{t} / h 2_{t}\right)-\ln \left(h 1_{0} / h 2_{0}\right)$, where $w$ is the competitive ability of either host genotype $\mathrm{C} 1$ or $\mathrm{C} 2(h l)$ relative to its competitor ( $h 2)$ and $h 1_{0}, h 2_{0}, h 1_{t}$, and $h 2_{t}$ are their frequencies at days 0 and $t$, respectively. $\ln$ is the natural logarithm. Because the change in frequency is divided by the number of days during which the hosts were competed against each other, competitive ability $w$ is given per day.

Experiment 2: Infection intensity in single hosts

Single newborn animals $(<24 \mathrm{~h})$ of all twelve host genotypes were placed individually in $1.5 \mathrm{~mL}$ medium and exposed to a single dose of 5,000 parasite spores. Host genotypes 
were replicated eight times yielding a total of 96 animals. Hosts were fed daily with $1.5 \times 10^{6}$ algal cells until day four and $2 \times 10^{6}$ algal cells thereafter. Newborns were removed daily and animals were placed into new medium on day eight of the experiment. On day 17, the experiment was terminated. We used quantitative PCR (qPCR) to detect infections and measured their intensity in individual hosts (Refardt and Ebert 2006). Primers 5'-GTAGTTGAAGTTCGGATGAGGAC-3' and 5'-CGGGACACCTCGCTAA AG- $3^{\prime}$ were used to amplify a 105 bp sequence from a variable region in the $16 \mathrm{~S}$ rRNA gene of the parasite. Infection intensity was quantified relative to DNA extracted from a spore suspension of known concentration and hence is given in spore equivalents per animal, an estimate of the total number of parasites in the host (Refardt and Ebert 2006).

Experiment 3: Parasite persistence in single-clone host populations

We measured parasite fitness as persistence of the infection in small single-clone host populations over 6-8 host generations. Populations consisted of a single clone of each of twelve host genotypes and were established by placing five adult hosts in a $100-\mathrm{mL}$ jar filled with $80 \mathrm{~mL}$ medium. Experimental populations of each clone were replicated eight times (96 populations total). To minimize variation due to infection success, populations were repeatedly exposed to parasite spores (day 3: 1,000 spores/jar, day 5: 1,500 spores/ jar, day 6: 1,500 spores/jar, day 24: 6,000 spores/jar). Populations were fed three times a week with $1.5 \times 10^{7}$ algae cells and water was changed during days 64-67. The experiment was terminated during days 115-124. The biggest animal from every experimental population was dissected and examined for signs of infection (phase contrast microscope, $400 \times$ magnification) in a random sampling order. Checking a single adult animal to infer parasite presence in a population is a reliable method because infection intensity increases with age and the oldest and biggest animals are the main carriers of the parasite load in a population (Stirnadel and Ebert 1997; Ebert 2005; Refardt and Ebert 2007).

\section{Statistical analysis}

Statistical tests were carried out with JMP 8 (SAS Institute Inc. 1989). Data from the competition experiment were analyzed with a nested, fully factorial ANOVA (Table 1, dependent variable: host competitive ability; factors: genotype of the host from Cumnor ('competitor 1', 2 levels), origin of the competitor ('origin competitor 2', 3 levels), genotype of the competitor ('competitor 2', 7 levels, nested in 'origin competitor 2'), and presence/absence of the parasite in the competition ('infection', 2 levels). To explore all effects in detail as well as in a more general context, a fixed effects and a mixed model (all factors except 'parasite' were treated as random effects) were employed. Kruskal-Wallis tests were used where the data did not satisfy assumptions of parametric tests.

Testing for a relationship between host competitive ability and parasite persistence

Using data from experiments 1 (host competition) and 3 (parasite persistence), we asked whether a relationship could be established between fitness components of the host (competitive ability) and the parasite (persistence). More specifically, we tested whether the change in competitive ability that occurred upon addition of the parasite was related to the relative persistence of the parasite in the two competing hosts. To this end, we established two variables for each of the 14 host pairs that were used in experiment 1: The 
Table 1 ANOVA table summarizing how the identity of two competing Daphnia magna genotypes (competitor 1, competitor 2 nested in origin of competitor 2), the infection status of the competitors, and all interactions contribute to the outcome of competition (Fig. 1)

\begin{tabular}{|c|c|c|c|c|c|c|c|c|c|c|}
\hline \multirow[t]{3}{*}{ Source } & \multicolumn{6}{|c|}{ Mixed model } & \multicolumn{4}{|c|}{ Fixed effects model } \\
\hline & \multicolumn{2}{|c|}{ Numerator } & \multicolumn{2}{|c|}{ Denominator } & \multirow[t]{2}{*}{$\mathrm{F}$} & \multirow[t]{2}{*}{$\mathrm{P}$} & \multirow[t]{2}{*}{ df } & \multirow[t]{2}{*}{ SS } & \multirow[t]{2}{*}{$\mathrm{F}$} & \multirow[t]{2}{*}{$\mathrm{P}$} \\
\hline & df & MS & df & MS & & & & & & \\
\hline Competitor 1 & 1 & 0.00403 & 1.37 & 0.00106 & 3.80 & 0.25 & 1 & 0.00403 & 40.86 & $<0.0001$ \\
\hline Origin competitor 2 & 2 & 0.00738 & 3.30 & 0.00292 & 2.53 & 0.22 & 2 & 0.00738 & 74.92 & $<0.0001$ \\
\hline Competitor 2 (origin comp. 2) & 4 & 0.00172 & 5.41 & 0.00081 & 2.13 & 0.21 & 4 & 0.00172 & 17.46 & $<0.0001$ \\
\hline Infection & 1 & 0.00056 & 2.96 & 0.00269 & 0.21 & 0.68 & 1 & 0.00056 & 5.66 & 0.0197 \\
\hline Comp. $1 \times$ origin comp. 2 & 2 & 0.00019 & 3.75 & 0.00016 & 1.19 & 0.40 & 2 & 0.00019 & 1.94 & 0.15 \\
\hline $\begin{array}{l}\text { Comp. } 1 \times \text { comp. } 2 \\
(\text { origin comp. } 2)\end{array}$ & 4 & 0.00016 & 4.00 & 0.00003 & 6.04 & 0.055 & 4 & 0.00009 & 1.61 & 0.18 \\
\hline Comp. $1 \times$ inf. & 1 & 0.00090 & 2.04 & 0.00003 & 32.05 & 0.028 & 1 & 0.00090 & 9.11 & 0.0034 \\
\hline Origin comp. $2 \times$ inf. & 2 & 0.00184 & 4.00 & 0.00068 & 2.69 & 0.18 & 2 & 0.00184 & 18.63 & $<0.0001$ \\
\hline $\begin{array}{l}\text { Comp. } 2 \times \text { inf. } \\
(\text { origin comp. } 2)\end{array}$ & 4 & 0.00068 & 4.00 & 0.00003 & 25.74 & 0.004 & 4 & 0.00068 & 6.87 & $<0.0001$ \\
\hline $\begin{array}{l}\text { Comp. } 1 \times \text { origin } \\
\text { comp. } 2 \times \text { inf. }\end{array}$ & 2 & 0.00003 & 3.84 & 0.00003 & 1.08 & 0.42 & 2 & 0.00003 & 0.29 & 0.75 \\
\hline $\begin{array}{l}\text { Comp. } 1 \times \text { comp. } 2 \times \\
\text { inf. (origin comp. } 2 \text { ) }\end{array}$ & 4 & 0.00003 & 83 & 0.00010 & 0.27 & 0.90 & 4 & 0.00003 & 0.27 & 0.90 \\
\hline Error & 83 & 0.00010 & & & & & 83 & 0.00010 & & \\
\hline
\end{tabular}

The analysis has been performed twice and both a mixed and a fixed effects model have been fitted. All competition assays were replicated four times, yet because one replicate was lost, degrees of freedom of the error term are only 83

difference of parasite persistence between the two host genotypes that competed against each other (measured in experiment 3), and the difference in the outcome of competition with and without parasite (measured in experiment 1). These variables were calculated relative to the Cumnor host genotype in the pair: a positive difference in persistence indicates that the parasite persists better in the Cumnor host genotype compared to its competitor. A positive change in competitive ability indicates that competitive ability of the Cumnor host genotype increased upon the addition of the parasite. A non-parametric rank correlation (Kendall's $\tau$ ) was used to explore the relationship between the variables.

\section{Results}

Experiment 1: Competition between hosts with and without infection

We performed competition assays in experimental populations consisting of two clones of D. magna. Genotypes $\mathrm{C} 1$ and $\mathrm{C} 2$ were competed against each of seven other host genotypes both in presence and in absence of the parasite. This revealed significant variation in competitive ability among these genotypes that was further influenced by their infection status (Fig. 1). A fixed effects ANOVA explained a large amount of this variation (Table 1, $r^{2}=81 \%$ ), which was mainly attributable to differences among the Daphnia genotypes and their origin $\left(r^{2}=60 \%\right)$. Overall, genotypes $\mathrm{C} 1$ and $\mathrm{C} 2$ suffered slightly more from an 


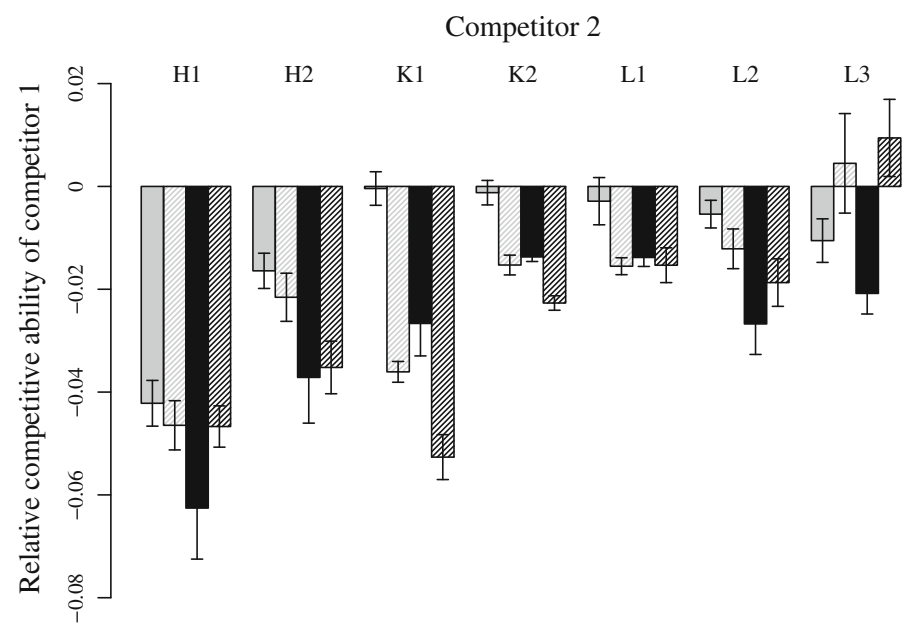

Fig. 1 Relative competitive ability of Daphnia magna genotypes C1 (gray) and C2 (black) as measured in competition against each of seven competitors. All competitions have been carried out with healthy (full bars) and infected individuals (striped bars). All competitions have been replicated four-fold. Error bars are 1 SEM

infection than their competitors (significant main effect of infection, $r^{2}=1 \%$ ). A further significant amount of variation was explained by those two-way interactions that included the factor 'infection' $\left(r^{2}=17 \%\right)$. Interactions between Daphnia genotypes and/or their origin were not significant and neither were three-way interactions $\left(r^{2}=3 \%\right)$.

Taken together, these results indicate that variation in competitive ability has a strong genetic component. Each Daphnia genotype can be assigned a specific competitive ability (significant main effects 'competitor 1', 'competitor 2' and 'origin of competitor 2'), which then also responds specifically to infection (interaction of these main effects with 'infection'), and determines the outcome of competition to a large degree. The absence of any significant genotype $\times$ genotype interactions between the two competitors further suggests that these individual traits are sufficient to determine the outcome of competition.

While the above analysis allowed us to identify exactly how individual factors contributed to the outcome of competition, their role must also be considered in natural populations, taking into account that variation among and within them is to be expected. To this end, the above analysis was repeated, this time by treating all factors except the infection status as random effects (Table 1). This revealed that variation due to main effects is within the range that is to be expected when a larger number of populations is sampled and confirmed that the differential response of Daphnia genotypes to infection is a key determinant for the outcome of competition, which explains $22 \%$ of the total variance.

The results indicate that the outcome of competition is shifted by an infection and this change is due to specific responses of the competing Daphnia genotypes. We therefore sought to understand what distinguishes these host genotypes and whether differences can be related to a differential performance of the parasite in these host genotypes.

Experiment 2: Infection intensity in single hosts

Five of 96 animals $(5.2 \%)$ died during the course of the experiment. In 25 of the remaining 91 animals, no parasite DNA was detected at the end of the experiment. Infection 


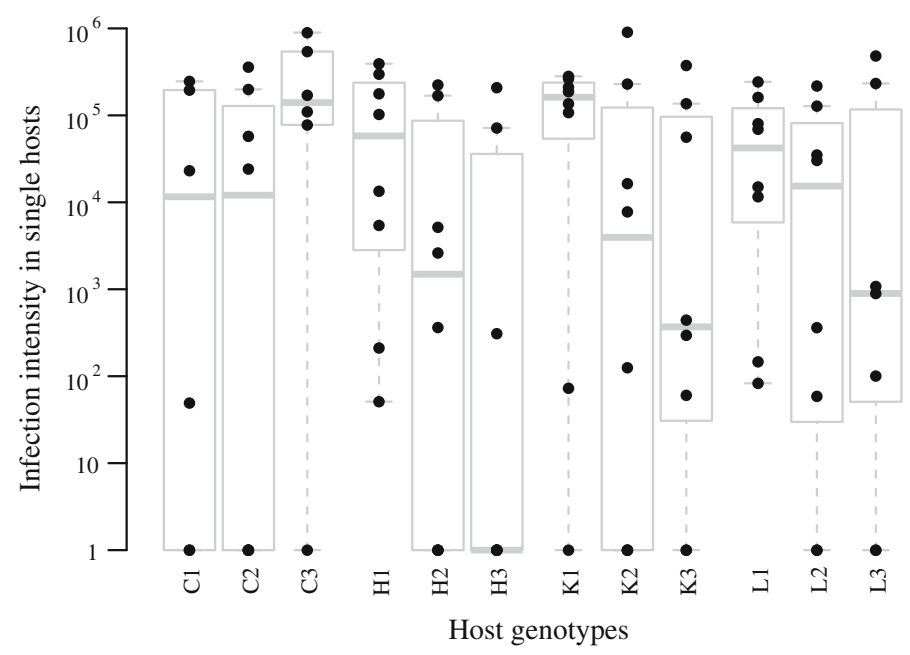

Fig. 2 Infection intensity as measured in single individuals of twelve Daphnia magna genotypes 17 days post infection (8 replicates each). To include data where no infection was detected, data shown are infection intensities +1 . To facilitate comparison of genotypes, box-plots are added

intensities varied widely (Fig. 2) but without any discernible differences that could be attributed to the Daphnia genotypes (Kruskal-Wallis $\chi^{2}=12.16, d f=11, P=0.35$ ) or their origin (Kruskal-Wallis $\chi^{2}=0.92, d f=3, P=0.82$ ). Because of its strong variation within genotypes and the absence of any effect that was attributable to differences among them, infection intensity was not considered for further analysis.

Experiment 3: Parasite persistence in single-clone host populations

Parasite persistence varied significantly among the twelve host genotypes (Fig. 3, KruskalWallis $\left.\chi^{2}=33.9, d f=11, P=0.0004\right)$. The observed variation is largely due to differences among the host genotypes and not due to differences among the populations they come from. Indeed, when data are grouped by host origin, variation in persistence is only marginally significant despite the higher number of replicates per factor level (KruskalWallis $\chi^{2}=8.7, d f=3, P=0.033$ ). We then proceeded to explore the relationship between competitive ability and parasite persistence.

Testing for a relationship between host competitive ability and parasite persistence

The change of competitive ability in response to infection (as estimated in the competition assays in experiment 1) was negatively correlated with the differential persistence of the infection (measured in experiment 3) in the two competing genotypes (Fig. 4, $n=14$, Kendall's $\tau=-0.67, P=0.0014)$. The host in which the infection had a lower persistence gained an advantage, with the size of this advantage being proportional to the differential persistence of the parasite in the two competitors.

When Figs. 1 and 4 are compared, further observations are noteworthy. First, host genotypes $\mathrm{C} 1$ and $\mathrm{C} 2$ (competitor 1) are weak competitors (most bars point downwards in Fig. 1). This is also evident in Fig. 4, where the change in fitness only becomes positive for a differential persistence of at least -0.4 , which means that these two genotypes require a 


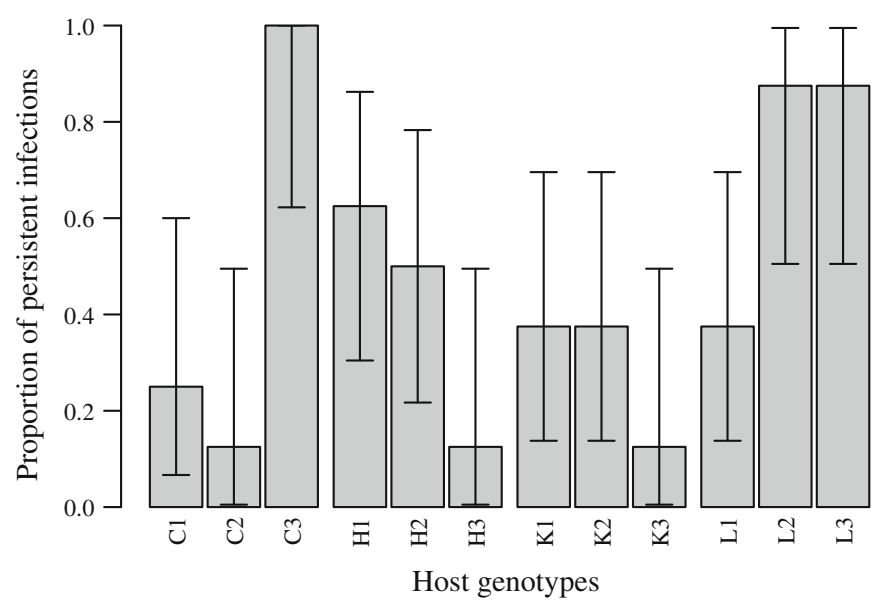

Fig. 3 Proportion of single-clone host populations (eight replicates each) in which infection persisted over at least six host generations. 95\% confidence intervals were calculated with the adjusted Wald method

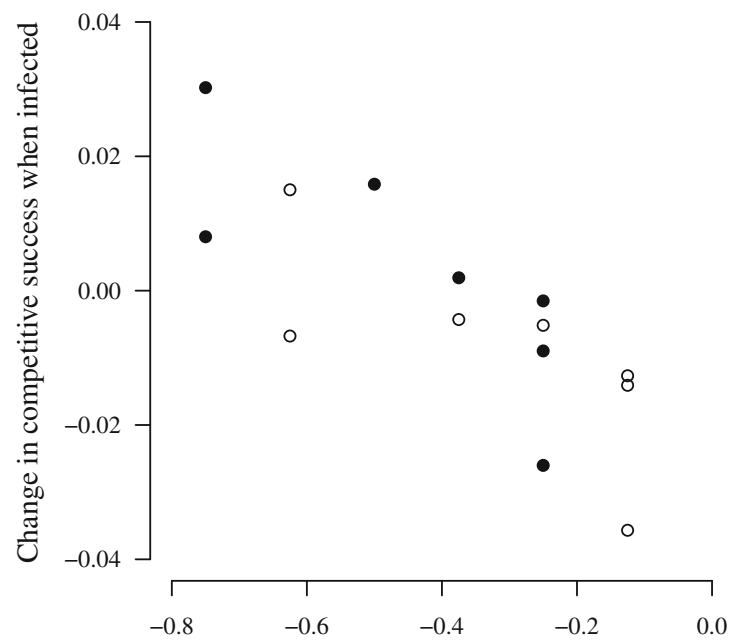

Difference of parasite persistence in the two competitors

Fig. 4 The change in the outcome of host competition that is caused by the addition of the parasite is related to the differential persistence of the parasite in the competitors. The plot contains data from experiments 3 (parasite persistence, $x$-axis) and 1 (host competition, $y$-axis): The $x$-axis shows the difference of parasite persistence between the competitors. Negative values indicate that the parasite persists better in competitor 2 , positive values indicate that the parasite persists better in competitor 1 . The $y$-axis shows the change in competitive success in the presence of the parasite that is experienced by competitor 1 (open circles: genotype $\mathrm{C} 1$; closed circles: genotype $\mathrm{C} 2$ ) as measured against seven different competitors. Negative values indicate that its competitive success declines when the parasite is present during competition, positive values indicate that its competitive success improves

substantially better parasite persistence in their competitor to obtain a net benefit in competition. Second, although differences in parasite persistence are related to changes in the outcome of competition (Fig. 4), they may not be sufficient to actually reverse its outcome (only a few bars change sign in Fig. 1). This indicates that the absolute 
differences in competitive ability among the host genotypes are generally larger than changes induced by this parasite.

\section{Discussion}

The relationship between host and parasite fitness is a key parameter to understand the ecology and evolution of infectious disease. Although many traits of parasites and hosts suggest that there is a negative relationship, its unequivocal inference is challenging (Hochberg 1998; Ebert and Bull 2003). We have obtained independent estimates of host and parasite fitness for a gut microparasite and several genotypes of its host, the freshwater crustacean Daphnia magna, and found that infection alters the competitive hierarchy of different host genotypes subtly but consistently. Moreover, we found evidence that the direction of parasite-induced change is related to the differential persistence of the infection in competing hosts. In the presence of the parasite, those host genotypes in which the infection persists less well gain an advantage when competing against genotypes in which the infection persists better.

The approach to measuring fitness components used here is novel and differs from the approach typically taken to characterize host-parasite interactions. Rather than focusing on individual host (life history) traits or a single step in the parasite's life cycle (e.g. infection or within-host growth), we attempted to obtain fitness estimates that encompass as many of these measures as possible. This was done with the aim of being able to make predictions about parasite-mediated selection in host populations while still being able to discern genotype-specific differences. We approximated the conditions in a natural population of Daphnia during the summer season, where hosts frequently compete under food-limitation and where infection by parasites is abundant. The approach required us to sacrifice a more detailed mechanistic insight, but allowed us to uncover a very subtle but highly specific host-parasite interaction. Equally, we had to limit ourselves to a single parasite isolate, which therefore confines the insights to the genetic variation on the side of the host.

Microparasites play an important role in the ecology and evolution of Daphnia (reviewed in Ebert 2005, 2008) and our study links the results that have been obtained by others. At the individual level, traits such as infectivity, parasite load, and resistance depend on the host genotype and the parasite isolate, as well as a genetic interaction between them (Carius et al. 2001; Decaestecker et al. 2003; Haag et al. 2003; Refardt and Ebert 2007). At the population level, parasites have been found to alter the evolutionary trajectory of host populations (Capaul and Ebert 2003; Haag and Ebert 2004; Mitchell et al. 2004), and resistance in natural populations can evolve in response to an epidemic (Duncan et al. 2006; Duffy and Sivars-Becker 2007). Here, we are able to demonstrate how variation at the individual level can translate into an evolutionary response at the population level, which confirms an important assumption that is implicit to our understanding of hostparasite interaction yet is rarely tested: the direction in which host fitness changes upon infection is negatively related to parasite fitness in the respective host genotypes.

A central question in disease ecology is whether parasites influence the dynamics of host populations (Tompkins and Begon 1999). There is considerable variation in virulence, with some parasite species driving host populations to extinction while the negative effect of others is difficult to detect (Stirnadel and Ebert 1997; Ebert et al. 2000; Decaestecker et al. 2005). Because there is also variation in host competitive ability (Haag et al. 2003), parasites with a low virulence may cause slight shifts in their host's fitness yet will not alter the main evolutionary route that a population takes (Capaul and Ebert 2003). 
Here, we observe such a case. The gut parasite that was used in this study has a low virulence. In laboratory experiments, infection had no measurable effect on host mortality and reduced host fecundity by only $15 \%$ (Decaestecker et al. 2003). This comparatively small reduction appears to be measurable only under controlled conditions, because a survey of natural populations found no differences in fecundity between infected and uninfected animals (Decaestecker et al. 2005). Similar to the observations made by Capaul and Ebert (2003), we find that the parasite alters the dynamics of host genotype frequencies but rarely the outcome of competition. However, by correcting for the variation in competitive ability among hosts, we can recover the relationship between host and parasite fitness that is hidden underneath.

The observation of such subtle effects raises the question of whether they are relevant in natural populations and whether parasite-induced changes have an effect that goes beyond the host population itself. Daphnia are important primary consumers in many aquatic ecosystems and a key link in aquatic food webs. In this context, two mechanisms have been discussed by which parasitism may cause shifts in the trophic cascade, selective predation of infected hosts (Packer et al. 2003) and the influence of disease on primary producers (Duffy 2007). Both require strong effects by the parasite to operate. To cause selective predation, disease must increase host vulnerability either by debilitation or a change in the visual appearance, and to change herbivore pressure by the host, disease must lower host population density. The parasite used here has an asymptomatic infection, and its low virulence makes it doubtful whether its sole action is sufficient to regulate host population density.

The implications may be different though. The parasite used here is widespread and occurs in most populations of D. magna that have been sampled throughout continental Europe and the UK (D. R. and D. E., unpublished results). Where data are available, the parasite is found at a consistently high prevalence across seasons (Decaestecker et al. 2005). Together, these data suggest that this is a very common parasite of $D$. magna, which possibly plays a key role in structuring the zooplankton community. Furthermore, the parasite used here is part of a larger number of horizontally transmitted gut parasites in D. magna, all of which are distinguished by their low virulence and inconspicuous pathology (Refardt et al. 2002). Interestingly, despite the apparently low selective pressure that they exert on their host, available data suggest that infection by any of them is determined by specific genotype $\times$ genotype interactions (this study, unpublished results, Refardt and Ebert 2007). Here, we show that these interactions can have fitness consequences for both antagonists. Daphnia populations typically harbor many parasites (Stirnadel and Ebert 1997; Decaestecker et al. 2005; Green 1974) and it will be important to assess their combined effect to fully understand host-parasite dynamics in natural populations. While virulent parasites are typically thought to be driving these dynamics, it may be the inconspicuous ones that tip the scales.

Acknowledgments We thank N. Basieux, J. Hottinger, S. Lass and L. Sygnarski for technical assistance, M. Kölliker for statistical advice, and H. K. Alexander for polishing the language. The manuscript benefitted from comments by J. Bull, J. Jokela, C. Kost, S. Lass, M. Zbinden, two anonymous reviewers, and the handling editor. The authors were supported by the Swiss National Science Foundation.

\section{References}

Anderson RM, May RM (1981) The population dynamics of microparasites and their invertebrate hosts. Phil Trans R Soc B 291:451-524

Anderson RM, May RM (1982) Coevolution of hosts and parasites. Parasitology 85:411-426 
Bull JJ (1994) Virulence. Evolution 48:1423-1437

Capaul M, Ebert D (2003) Parasite-mediated selection in experimental Daphnia magna populations. Evolution 57:249-260

Carius HJ, Little TJ, Ebert D (2001) Genetic variation in a host-parasite association: potential for coevolution and frequency-dependent selection. Evolution 55:1136-1145

Clarke BC (1979) Evolution of genetic diversity. Proc R Soc B 205:453-474

de Roode JC, Yates AJ, Altizer S (2008) Virulence-transmission trade-offs and population divergence in virulence in a naturally occurring butterfly parasite. Proc Natl Acad Sci USA 105:7489-7494

Decaestecker E, Vergote A, Ebert D, Meester LD (2003) Evidence for strong host clone-parasite species interactions in the Daphnia microparasite system. Evolution 57:784-792

Decaestecker E, Declerck S, Meester LD, Ebert D (2005) Ecological implications of parasites in natural Daphnia populations. Oecologia 144:382-390

Dieckmann U (ed) (2002) Adaptive dynamics of infectious disease. Cambridge University Press, Cambridge

Duffy MA (2007) Selective predation, parasitism, and trophic cascades in a bluegill-Daphnia-parasite system. Oecologia 153:453-460

Duffy MA, Sivars-Becker L (2007) Rapid evolution and ecological host-parasite dynamics. Ecol Lett $10: 44-53$

Duncan AB, Mitchell SE, Little TJ (2006) Parasite-mediated selection and the role of sex and diapause in Daphnia. J Evol Biol 19:1183-1189

Ebert D (2005) Ecology, epidemiology, and evolution of parasitism in Daphnia [Internet]. National Library of Medicine (US), National Center for Biotechnology Information, Bethesda, MD. Available from http://www.ncbi.nlm.nih.gov/books/NBK2036. (Accessed Feb 2011)

Ebert D (2008) Host-parasite coevolution: insights from the Daphnia-parasite model system. Curr Opin Microbiol 11:290-301

Ebert D, Bull JJ (2003) Challenging the trade-off model for the evolution of virulence: is virulence management feasible? Trends Microbiol 11:15-20

Ebert D, Zschokke-Rohringer CD, Carius HJ (1998) Within- and between-population variation for resistance of Daphnia magna to the bacterial endoparasite Pasteuria ramosa. Proc R Soc B 265:2127-2134

Ebert D, Lipsitch M, Mangin KL (2000) The effect of parasites on host population density and extinction: experimental epidemiology with Daphnia and six microparasites. Am Nat 156:459-477

Ebert D, Haag C, Kirkpatrick M, Riek M, Hottinger J, Pajunen VI (2002) A selective advantage to immigrant genes in a Daphnia metapopulation. Science 295:485-488

Fels D (2005) The effect of food on microparasite transmission in the waterflea Daphnia magna. Oikos 109:360-366

Fels D, Lee VA, Ebert D (2004) The impact of microparasites on the vertical distribution of Daphnia magna. Arch Hydrobiol 161:65-80

Frank SA (1996) Models of parasite virulence. Quart Rev Biol 71:37-78

Grech K, Watt K, Read AF (2006) Host-parasite interactions for virulence and resistance in a malaria model system. J Evol Biol 19:1620-1630

Green J (1974) Parasites and epibionts of Cladocera. Trans Zool Soc Lond 32:417-515

Haag CR, Ebert D (2004) Parasite-mediated selection in experimental metapopulations of Daphnia magna. Proc R Soc B 271:2149-2155

Haag CR, Sakwinska O, Ebert D (2003) Test of synergistic interaction between infection and inbreeding in Daphnia magna. Evolution 57:777-783

Hamilton WD (1980) Sex versus non-sex versus parasite. Oikos 35:282-290

Hamilton WD, Zuk M (1982) Heritable true fitness and bright birds: a role for parasites? Science 218:384-387

Hartl DL (1987) A primer of population genetics. Sinauer, Sunderland

Hochberg ME (1998) Establishing genetic correlations involving parasite virulence. Evolution 52:1865-1868

Jensen KH, Little TJ, Skorping A, Ebert D (2006) Empirical support for optimal virulence in a castrating parasite. PLoS Biol 4:e197

Jungen H, Hartl DL (1979) Average fitness of populations of Drospohila melanogaster as estimated using compound-autosome strains. Evolution 33:359-370

Larsson JIR, Ebert D, Vávra J, Voronin VN (1996) Redescription of Pleistophora intestinalis Chatton, 1907, a microsporidian parasite of Daphnia magna and Daphnia pulex, with establisment of the new genus Glugoides (Microspora, Glugeidae). Europ J Protistol 32:251-261

Little TJ, Carius HJ, Sakwinska O, Ebert D (2002) Competitiveness and life-history characteristics of Daphnia with respect to susceptibility to a bacterial pathogen. J Evol Biol 15:796-802 
Mackinnon MJ, Read AF (1999) Genetic relationships between parasite virulence and transmission in the rodent malaria Plasmodium chabaudi. Evolution 53:689-703

May RM, Anderson RM (1983) Epidemiology and genetics in the coevolution of parasites and hosts. Proc R Soc B 219:281-313

Mitchell SE, Read AF, Little TJ (2004) The effect of a pathogen epidemic on the genetic structure and reproductive strategy of the crustacean Daphnia magna. Ecol Lett 7:848-858

Packer C, Holt RD, Hudson PJ, Lafferty KD, Dobson AP (2003) Keeping the herds healthy and alert: Implications of predator control for infectious disease. Ecol Lett 6:797-802

Refardt D, Ebert D (2006) Quantitative PCR to detect, discriminate and quantify intracellular parasites in their host: an example from three microsporidians in Daphnia. Parasitology 133:11-18

Refardt D, Ebert D (2007) Inference of parasite local adaptation using two different fitness components. J Evol Biol 20:921-929

Refardt D, Canning EU, Mathis A, Cheney SA, Lafranchi-Tristem NJ, Ebert D (2002) Small subunit ribosomal DNA phylogeny of microsporidia that infect Daphnia (Crustacea: Cladocera). Parasitology 124:381-389

Rigby MC, Hechinger RF, Stevens L (2002) Why should parasite resistance be costly? Trends Parasitol $18: 116-120$

Rolff J, Joop G (2002) Estimating condition: pitfalls of using weight as a fitness correlate. Evol Ecol Res 4:931-935

Roy BA, Kirchner JW (2000) Evolutionary dynamics of pathogen resistance and tolerance. Evolution 54:51-63

SAS Institute Inc (1989) JMP, Version 8. SAS Institute Inc., Cary

Schmid-Hempel P, Ebert D (2003) On the evolutionary ecology of specific immune defence. Trends Ecol Evol 18:27-32

Schwarzenbach GA, Ward PI (2006) Responses to selection on phenoloxidase activity in yellow dung flies. Evolution 60:1612-1621

Stearns SC (1992) The evolution of life histories. Oxford University Press, Oxford

Stirnadel HA, Ebert D (1997) Prevalence, host specificity and impact on host fecundity of microparasites and epibionts in three sympatric Daphnia species. J Anim Ecol 66:212-222

Thompson JN (1994) The coevolutionary process. University of Chicago Press, Chicago

Tompkins DM, Begon M (1999) Parasites can regulate wildlife populations. Parasitol Today 15:311-313

Vijendravarma RK, Kraaijeveld AR, Godfray HCJ (2009) Experimental evolution shows Drosophila melanogaster resistance to a microsporidian pathogen has fitness costs. Evolution 63:104-114 